ARTíCULOS DE INVESTIGACIÓN

\title{
La clase invertida o flipped classroom: Intervención didáctica en una educación virtual de emergencia
}

\author{
A aula invertida ou flipped classroom: A intervenção didática \\ em uma educação virtual de emergência
}

The flipped classroom: Didactic intervention in a virtual emergency education

\author{
Mauricio Andrés Figueroa Mendoza \\ Universidad Santo Tomás, Chile
}

RESUMEN Este trabajo expone una experiencia de intervención didáctica que se implementó con el fin de afrontar lo que el nuevo escenario virtual, provocado por la emergencia sanitaria, impuso en la continuidad del proceso educativo. La intervención didáctica consistió en la aplicación del modelo de aula invertida o flipped classroom en el curso de Introducción al Derecho Civil, durante el segundo semestre de 2020. El desarrollo de este trabajo consta de tres partes: la primera aborda los antecedentes que obligan a la modificación del proceso educativo y el cambio de contexto o escenario al que se enfrenta la educación; en la segunda se identifican los principales aspectos y las principales ventajas del método de clase invertida en la enseñanza superior; y en la tercera se describe la intervención didáctica realizada en el curso de primer año. Además, se exponen los resultados obtenidos en la aplicación de una encuesta de satisfacción realizada a los estudiantes que participaron de esta experiencia.

PALABRAS CLAVE Pandemia, clase invertida, intervención didáctica, introducción al derecho civil, metodología educativa, proceso educativo.

RESUMO Neste trabalho é exposta uma experiência de intervenção didática, que foi implementada para enfrentar o que o novo cenário virtual, provocado pela emergência de saúde, impõe à continuidade do processo educativo. A intervenção didática consiste na aplicação do modelo de sala de aula invertida ou sala invertida na disciplina de Introdução ao Direito Civil, durante o segundo semestre do ano de 2020. O desenvolvimento deste trabalho consta de três partes: a primeira aborda os antecedentes que o requerem a modificação do processo educacional e a mudança de contexto ou cenário que a educação enfrenta; em segundo lugar, são identificados os principais aspectos, juntamente 
com as vantagens do método da aula invertida no ensino superior; enquanto a terceira parte descreve a intervenção didática realizada no curso de primeiro ano, além de expor os resultados obtidos na aplicação de uma pesquisa de satisfação realizada com os alunos que participaram da experiência.

PALAVRAS-CHAVE Pandemia, intervenção didática de classe invertida, introdução ao direito civil, metodologia educacional, processo educacional.

\begin{abstract}
In this work an experience of didactic intervention is exposed, which was implemented to face what the new virtual scenario, caused by the health emergency, imposes on the continuity of the educational process. The didactic intervention consists of the application of the inverted classroom model or flipped classroom in the course of Introduction to Civil Law, during the second semester of the year 2020. The development of this work consists of three parts: the first addresses the antecedents that require the modification of the educational process and the change of context or scenario that education faces; secondly, the main aspects are identified, together with the advantages of the flipped class method in higher education; while the third part describes the didactic intervention carried out in the first year course, in addition to exposing the results obtained in the application of a satisfaction survey carried out on the students who participated in the experience.
\end{abstract}

KEYWORDS Pandemic, flipped classroom, didactic intervention, introduction to civil law, educational methodology, educational process.

\title{
Antecedentes de cambio
}

Durante 2020, la humanidad fue testigo de una pandemia mundial, esta vez generada por el covid-19, que, junto con activar todas aquellas consecuencias ligadas a la salubridad de los Estados afectados, trajo consigo una serie de modificaciones en los estándares de vida, en las relaciones personales y en aquellos cánones que permanecieron por mucho tiempo arraigados en las diversas sociedades.

Como ocurre en los procesos de transformación, la pandemia trajo una serie de cambios de distintas naturaleza e intensidad, lo que, sin duda, nos hace pensar en un evento causal de dimensiones históricas. Así, en el caso de la educación, se tuvieron que adoptar tendencias existentes, pero no masificadas en la práctica, como las metodologías activas, las evaluaciones en línea, la implementación de comunidades virtuales, los cambios en la transmisión del conocimiento y los paradigmas comunicativos en educación, entre otros (Bartolomé-Pina, 2020: 14).

Un primer efecto provocado por la pandemia del covid-19 fue la obligación de los planteles de educación superior a suspender las clases presenciales y volcar la práctica a escenarios virtuales, lo que podría enmarcarse dentro de un cambio contextual o 
de escenario. Lo anterior, con todas las dudas posibles y bajo un manto de incógnitas que inundan y hacen desaparecer las certezas que hasta los docentes más experimentados tenían hasta antes de la pandemia (García-Peñalvo, 2020: 2-3).

Uno de los grandes desafíos fue darle continuidad al servicio educacional, lo que se tradujo en una importante inversión de recursos financieros, tecnológicos y humanos, en especial cuando la tendencia a mantener espacios virtualizados o híbridos comenzó a tomar mayor peso en las diversas instituciones. Así, llegó a plantearse, casi como un desafío a futuro, la implementación de sistemas b-learning en la educación superior.

Otra de las situaciones que debieron afrontar, esta vez, los actores de la educación, como los profesores, fue transformar la docencia presencial en una docencia en línea, a través de una pantalla, en tiempo real y con todas las deficiencias en el conocimiento y en el manejo de las tecnologías. Esto trasformó el desafío en una oportunidad y en una posibilidad de explorar herramientas digitales que, hasta ese momento, eran desconocidas para muchos. Este contexto obligó a todos a generar el contenido necesario para reactivar su práctica docente (Carranza y Zamora, 2020: 168).

La ampliación tecnológica constituyó un desafío en todo nivel, ya que las barreras digitales no solo plantearon un problema para los docentes en términos de conocimiento y manejo de tecnologías de la información y comunicación, sino que el problema se amplió incluso a los estudiantes, quienes encontraron importantes desafíos a la hora de incorporarse al nuevo contexto digital, como el manejo de plataformas digitales que harían las veces de aula y centro de recursos o la no disposición de los recursos tecnológicos, fueran dispositivos o una conexión a internet que les permitiera conectarse a los escenarios virtuales y asistir a la sesión de clases o disponer de los materiales de apoyo. En este escenario, la Cepal y la Unesco (2020: 7) señalaron que:

Es preciso entender estas brechas desde una perspectiva multidimensional, porque no se trata solo de una diferencia de acceso a equipamiento, sino también del conjunto de habilidades que se requieren para poder aprovechar esta oportunidad, que son desiguales entre estudiantes, docentes y familiares a cargo del cuidado y la mediación de este proceso de aprendizaje que hoy se realiza en el hogar. Por lo mismo, es central que las políticas de promoción de un acceso más igualitario a la tecnología comiencen por reconocer estas diferentes dimensiones que estructuran las desigualdades sociales en la región y trabajar intencionalmente para revertirlas.

\section{Problemas de fondo a resolver en la educación en situación de pandemia}

Para afrontar los desafíos que planteó la emergencia sanitaria, que sigue siendo una exigencia en la actualidad, se implementó un plan de acción que debió, necesariamente, centrarse en la priorización de contenidos que permitiera determinar el contenido de la lección en conjunto con los aprendizajes esperados, además de la flexibilización 
en la entrega de evidencias por parte del estudiante, junto con la comunicación y la retroalimentación de los aprendizajes, cuestiones que se transformaron en un primer bloque del cimiento educativo. Por otro lado, debe tenerse en cuenta que, frente a la virtualidad o semivirtualidad, que es lo que podría esperarse para el futuro, la gestión del tiempo adecuado para el aprendizaje debió y debe considerarse, a través de momentos sincrónicos y asincrónicos, en íntima relación con una metodología activoparticipativa que pueda alternar en ocasiones con una clase tradicional.

Otro de los desafíos planteados para la educación en tiempos de pandemia lo constituyó la búsqueda de estrategias que permitieran cumplir, a través de la virtualidad, con ámbitos tradicionales de la educación presencial, como el aspecto social de la educación, la dimensión cognitiva del proceso educativo y la didáctica en función de logros educacionales (Salinas, 2020: 18). En lo social, el desafío se plantea en la comunicación entre estudiante y docente, y en la detección de problemas asociados al entorno del alumnado en particular, entre otros. En lo cognitivo, se plantea la disyuntiva no solo de lo que deben aprender los estudiantes, sino del nivel de destreza que deben alcanzar, de modo de plantearse la posibilidad de una lección que le permita al educando alcanzar un nivel básico de habilidades, como lo es la comprensión o, por el contrario, avanzar hacia aquellas habilidades de orden superior.

Una vez que se decide por esta última opción, aparece la dimensión didáctica: ¿cómo lograr el aprendizaje en el nivel deseado? Lo que plantea una primera alternativa para el docente, por un lado, optar por un método tradicional de clases, lo que en el espacio universitario sería la clase magistral; o, por el contrario, optar por una metodología activo-participativa que asegure mantener la práctica habitual desarrollada en tiempos de normalidad. Todo esto, sin duda, plantea, a su vez, el desafío conexo de la participación activa del estudiantado, pero esta vez por vía remota o digital.

Lo anterior, sin desconocer que, pase lo que pase en el futuro, en el nuevo paradigma educativo todos los miembros de las distintas comunidades se han transformado en ciudadanos digitales (Salinas, 2020: 20) y, frente a ello, el punto de no retorno está determinado por la tecnología digital, sea de manera presencial o remota.

Los problemas descritos son un fiel reflejo de la precariedad del modelo de educación a distancia (Alcántara, 2020: 75), en tanto que la adaptación en aquellas instituciones, regiones o espacios en los que la educación a distancia se encuentra implementada, los problemas y las brechas, se redujeron al mínimo, frente al otro extremo, el que se tuvo que replantear la práctica y la instrucción.

\section{¿Qué y cómo enseñar bajo el nuevo escenario?}

Uno de los alcances que se debe tener en consideración en la educación superior, es que la formación del estudiante apunta a ciertas habilidades superiores de orden profesional y cuya finalidad es el desenvolvimiento pleno dentro de la sociedad a través 
de las competencias profesionales que han tributado al desarrollo de dichas competencias, lo que se reconoce, en términos generales, como alfabetización académica (Sánchez y otros, 2020).

Esto, necesariamente, obliga a un cambio de sentido y a seguir el camino dificultoso, ya sea en tiempos normales, ya sea en tiempos de virtualidad. Así lo expresan Lima y otros (2020: 8):

Una concepción más promisoria, que supera la concepción de la enseñanza como mera «transmisión de contenidos», la constituye el desarrollo de la capacidad de desempeño o conducta profesional. Cabe señalar que no existe una dicotomía entre estas dos concepciones, sino una relación integral, ya que el desarrollo de la conducta profesional implica la transmisión de contenidos. Sin embargo, va más allá, pues también requiere la crítica, el análisis y la sistematización del conocimiento existente, así como la integración entre conocimientos de diferentes áreas del conocimiento. El concepto de enseñanza caracterizado por el desarrollo de conductas profesionales, sin embargo, no se limita a permitir a los estudiantes reproducir conocimientos (resultado de procesos de enseñanza guiados por la transmisión de contenidos) o afrontar conocimientos, sino afrontar la realidad social. del conocimiento.

Atendida la necesidad de adoptar el desarrollo de habilidades profesionales por sobre la mera transferencia de contenidos, se plantea la situación particular de la estrategia didáctica para concretar el aprendizaje de los estudiantes, ya que, dependiendo de la estrategia, se presenta una mayor o menor flexibilidad y utilidad a la misma didáctica, lo que permite conectarlo con procesos de mayor interacción entre los estudiantes y el docente, y la conexión con el objeto de conocimiento. Es por ese motivo que la estrategia didáctica debe «ser seleccionada de forma que contribuyan al tratamiento de los contenidos de manera adecuada para que el aprendizaje significativo se logre» (Montes y otros, 2020: 246).

Al enfrentar el proceso de aprendizaje-enseñanza a través de una estrategia didáctica, lo que se produce, en un sentido amplio, es que para el logro de los aprendizajes se ponen a disposición de él una serie métodos, técnicas y medios de diversa naturaleza a fin de integrar cada uno de los componentes del proceso que permitan alcanzar la finalidad deseada (Jiménez y Robles, 2016: 108). En este sentido, las metodologías denominadas activo-participativas se presentan como una alternativa en la práctica educativa actual.

\section{Consideraciones en torno a la carrera de Derecho: Inicio de una decisión}

En el caso de la carrera de Derecho, si bien el fenómeno antes descrito afectó igualmente la práctica pedagógica, la manera de afrontarlo no puede haber estado exenta de análisis en torno a dos disyuntivas que tradicionalmente han sido parte de la discusión académica, la clase tradicional o la clase activa, la memorización de conte- 
nidos o desarrollo de habilidades profesionalizantes; planteamientos de los que ha habido cuantiosos aportes y de los que solo nos remitiremos a lo dicho por diversos autores nacionales a la hora de inclinarse por la clase activa; el desarrollo de habilidades superiores en estudiantes de derecho y metodologías activas como parte de la didáctica en la educación del derecho (Lazo, 2011; Solari, 2012; Aedo, 2014; Elgueta y Palma, 2015; Coloma, 2006; Benfeld, 2016; Figueroa, 2019).

Todo esto ante la necesidad de fortalecer algunos aspectos evaluados durante el primer semestre de 2020, los que debían mejorar en algunos puntos, como el rendimiento académico, la motivación de los estudiantes, la participación activa de los estudiantes en su proceso de formación y, finalmente, el aprovechamiento efectivo del tiempo de clase por parte del estudiante para fortalecer los conocimientos adquiridos.

Para cumplir con lo que denominamos como desafío RAMPA, del acrónimo RA (rendimiento académico), M (motivación), $\mathrm{P}$ (participación activa del estudiante) y A (aprovechamiento efectivo del tiempo), es que se propuso aplicar la metodología de «clase o aula invertida», para dar solución a los aspectos disminuidos en la formación de nuestros estudiantes.

\section{Clase invertida o flipped classroom}

\section{Generalidades sobre el método}

El flipped classroom o clase invertida encuentra sus antecedentes como concepto hacia el 2000, cuando Lage, Platt y Treglia comenzaron a trabajar con el término flipped (Andrade y Chacón, 2018: 252). Sin embargo, no fue sino hasta 2007 cuando el término comenzó a adquirir mayor solidez y masificación gracias a la tarea de Bergmann y Sams (2014: 18), quienes comenzaron a trabajar con los primeros videos ante la necesidad de acercar la instrucción a los estudiantes que no pudieron asistir a sus clases:

De este modo, nació nuestra «clase al revés». Nos propusimos grabar todas nuestras lecciones de Química y de Química Avanzada durante el año escolar 2007-2008. Para hacernos las cosas más fáciles, uno de nosotros desarrollaba la Unidad 1 de Química, y el otro la Unidad 1 de Química Avanzada. Luego intercambiábamos la siguiente. Esto significó madrugar muchas mañanas para Jonathan, el madrugador, y trasnochar muchas veces, para Aaron, el más trasnochador de nuestro dúo.

De esta manera, lo que comenzó como un experimento para estos profesores terminó siendo una gran ayuda para sus estudiantes, quienes vieron personalizada su educación a través de esta nueva metodología.

La clase invertida constituye, en su inicio, un modelo que apunta a que los estudiantes que no pueden asistir a clases no pierdan la lección impartida y logren recuperarla (Pérez, 2017: 86) e internalizarla de manera activa (Gómez y López, 2019), ya sea en sus tiempos fuera de clase o en la misma clase. 
El espacio físico tradicional de adquisición de conocimiento, como el aula, se transforma, deja de ser el único espacio posible y se abre a la posibilidad de que el hogar de cada estudiante se complemente con el aula. Además, en el último tiempo, en Chile se ha incorporado un nuevo escenario: el espacio virtual o la red virtual (Abad y González, 2019: 31). Así, es una propiedad interesante del flipped la apertura de nuevos escenarios de aprendizaje, autoaprendizaje y aprendizaje colaborativo.

El flipped classroom ha ido ganando cada vez mayor aceptación en diversas áreas de contenido y de formación, todo en atención a la calidad de los aprendizajes y el tiempo efectivo de clases (Tourón y otros, 2014), además de permitir un mayor acercamiento entre los estudiantes y el docente, mejorando y fortaleciendo la comunicación entre ellos (Martín y Santiago, 2016; Melo y Sánchez, 2017; Sánchez, 2017). Todo esto se ha traducido en un aumento en la calidad de las intervenciones, en la propia motivación que los estudiantes adquieren en su proceso de aprendizaje y en la gestión de sus conocimientos.

La metodología de clase invertida o flipped classroom corresponde a un modelo de estrategia educativa que permite innovar en la sala de clases y, particularmente, en el mecanismo de afianzamiento de los conocimientos (Bergmann y Sans, 2017). Es un modelo en el que los elementos de una clase se invierten y que apunta más allá del simple reordenamiento del trabajo en casa y el desarrollo de la clase (Sánchez y otros, 2017: 343), lo que conlleva a un reordenamiento de tareas y planificación bajo una misma práctica, mismos objetivos y mismos resultados. De esta manera, la clase invertida no solo busca invertir un orden de tareas o actividades, sino que busca desarrollar, en un número reducido de contenidos, un pensamiento en expansión que le permita al aprendiz la construcción de su propio conocimiento a través de habilidades de todo orden, de manera de hacer del estudiante el arquitecto y el constructor de su aprendizaje (Galindo-Domínguez y Bezanilla, 2019: 82).

De lo preceptuado hasta ahora, sin duda el método flipped classroom presenta ciertos pilares desde los que se debe abordar su práctica en el proceso educativo. La incorporación de la clase invertida debe estar sustentada en: i) la construcción de entornos flexibles de aprendizajes que beneficien al estudiante, además de la flexibilidad del docente ante el nuevo escenario; ii) modelo centrado en el estudiante, cuyo objetivo central es crear oportunidades de aprendizajes en el estudiante; iii) contenidos y actividades ad hoc, seleccionadas por el profesor, que permitan, a su vez, un mejor aprovechamiento del tiempo en el aula; y iv) docentes expertos con un alto sentido pedagógico para planificar, guiar y acompañar a los estudiantes, posibilitando la mayor eficiencia en torno al aprendizaje (Cobeña y Rodríguez, 2019: 6).

El orden de la instrucción consta de distintas etapas, pero, sin duda, el elemento diferenciador consiste en la disposición de los materiales de aprendizaje que el profesor acerca al estudiante antes de la clase (Moya y Soler, 2019: 1.227), ya sea para su lectura, observación o elaboración de alguna actividad, lo que, en un inicio, constitu- 
ye el primer indicio de exigencia del modelo, ya que, de una u otra forma, se requiere de un esfuerzo cognitivo y de una disposición al trabajo por parte del estudiante, a diferencia de la clase tradicional (Prieto y otros, 2018: 178), en la que solo requiere la atención inicialmente.

\section{Rol del docente en el modelo de clase invertida}

En el modelo tradicional de clase, el docente conduce el proceso con un importante protagonismo. Su rol activo muchas veces juega en contra del rol del mismo estudiante, quien alcanza un rol secundario en el proceso y solo adquiere participación en las evaluaciones, es decir, en la parte final de cada instrucción. El modelo de clase invertida instala, en la práctica docente, un rol distinto. Necesariamente debe mantener una participación activa, pero desde el rol de orientador, de facilitador del conocimiento, de experiencias y motivador de los estudiantes. Deja, por tanto, de ser un mero proveedor de información (Abad y González, 2019: 31).

Si bien el flipped es parte de las denominadas metodologías activo-participativas ello no obsta a que la clase tradicional o cátedra magistral, como se le conoce en los espacios universitarios, deba ser desechada por completo. Por el contrario, se reconoce la necesidad de mantenerlas para cumplir con el contenido teórico de la instrucción, en aquella medida en que su complejidad requiera de la habilidad pedagógica del docente para transformar lo difícil en una explicación cercana al estudiante.

El método de clase invertida no busca un cambio radical en el docente. Como lo plantean Abad y González (2019: 34), «no se pretende que las instituciones educativas y los profesores renuncien a sus responsabilidades, sino que orienten sus objetivos a actuar de guías de aprendizaje proporcionando las herramientas y estrategias necesarias al alumnado».

Tampoco implica que el método del flipped classroom disminuya la importancia del docente o le haga perder algún protagonismo en el proceso educativo, ni mucho menos, como se señaló, le permita delegar su trabajo a los estudiantes, ya que, necesariamente, se debe considerar una inversión importante de tiempo, no solo para construir el material, la actividad o el video, sino que el pensarlo, intencionarlo y planificarlo para el logro de aprendizajes significativos en los estudiantes. Es por ello que una buena inversión de tiempo debe ser, además, la que le permita al docente saber con exactitud qué es lo que quiere que sus estudiantes aprendan, qué serán capaces de realizar y en qué medida deberán aplicarlo (Rotellar y Cain, 2016: 3).

La tarea del docente implica acercar a los estudiantes los mejores recursos de aprendizaje, ser un guía para atender dudas e inquietudes y, sobretodo, acercar la teoría a la práctica (Perdomo, 2016: 4).

En cada texto, video o actividad práctica se debe intencionar (González y otros, 2017: 62) a partir de lo que se desea lograr a través del material. Debe, a su vez, ser 
consciente de lo que se busca lograr en el estudiante, ya sea como meta de aprendizaje o como experiencia pedagógica como medio idóneo para el aprendizaje (Mok, 2014: 7).

El docente debe asegurarse de que lo más importante en el proceso de aprendizaje-enseñanza se produzca en el aula, ya que debe controlar lo que se discute, se analiza, se evalúa, mientras que el resto de las tareas básicas se llevan al hogar del estudiante (Bergmann y Sams, 2014).

\section{Rol activo del estudiante}

El modelo de clase invertida contempla un cambio en el rol del aprendiz, desde una postura pasiva a una activa, de mayor involucramiento en el proceso educativo, por lo tanto, se desecha la idea de un estudiante como mero receptor (Albornoz y otros, 2020: 3). Así, se hace partícipe y responsable de su propio aprendizaje, en ocasiones gestionando el conocimiento, en otros momentos regulando sus tiempos y coordinando el trabajo con sus pares.

La primera tarea del estudiante corresponde a la visualización, la lectura o la elaboración, ya sea del texto, video o actividad que el profesor encarga como punto de partida, condición mínima o requerimiento básico para la clase, de manera que, en la sesión, el estudiante disponga de tiempo suficiente para solicitarle que pueda profundizar, explicar conceptos, despejar dudas o simplemente realizar otras actividades planteadas por el docente. De esta manera, el tiempo en clases se debe aprovechar de una manera distinta a la tradicional (Berenguer, 2016).

A diferencia del modelo tradicional o la clase magistral, en la clase invertida el rol del estudiante le permite ampliar su mirada y su desarrollo hacia habilidades de orden superior en plena clase. El analizar, aplicar, evaluar y sintetizar corresponden a tareas llevadas a cabo en presencia del docente, en actividades de clase, mientras que las habilidades de comprensión y memorización corresponden a tareas que el estudiante realiza fuera del aula, antes de llegar al encuentro con el docente y sus compañeros. De esta manera, el docente tiene la misión de planificar con tal prolijidad las actividades y las tareas de los estudiantes, que debe procurar que el trabajo tanto individual como grupal, sin la mediación del docente, sea de baja complejidad, mientras que las actividades o tareas de mayor complejidad deben realizarse en presencia del docente, con retroalimentación directa, constante e inmediata, espacio que coincide con una sesión sincrónica o directa. En ese sentido, debe tenerse en cuenta la estrecha relación entre la clase en presencia del docente y la oportunidad de complementar la teoría (como tarea personal del estudiante) y la práctica a través de la aplicación de los conocimientos (Andrade y Chacón, 2018: 255). 


\section{Beneficios del método de clase invertida}

Diversos estudios han planteado los beneficios de la clase invertida, los que por cierto son variados. Ellos cuentan desde el rendimiento académico, la comunicación entre estudiantes, y entre estudiantes y docentes, hasta el aumento de la motivación de los propios estudiantes por el aprendizaje (Gómez y López, 2019; Hinojo y otros, 2019) y la carrera que estudian.

El trabajo autónomo y colaborativo se ve favorecido en la práctica de la clase invertida. Esto, en atención a la flexibilidad y a la adaptabilidad del modelo, lo que, sin duda, permite que los estudiantes canalicen sus esfuerzos y encaminen su autoaprendizaje (Gámiz y Gallego, 2016; Abeysekera y Dawson, 2015).

Si bien el éxito de los aprendizajes habría estado centrado de manera inconexa en el rol del docente y del estudiante, el flipped classroom requiere de un esfuerzo en conjunto, entrelazado por el esfuerzo de ambas partes, en tanto el docente entrega el material propuesto, el estudiante debe trabajarlo, estudiarlo y darle la dedicación necesaria para alcanzar el aprendizaje profundo (Andrade y Chacón, 2018: 257).

Así, una de las importantes deficiencias detectadas en las aulas de derecho tiene relación con el desarrollo del pensamiento crítico, el razonamiento lógico y jurídico, junto con otras habilidades que transitan en niveles cognitivos superiores. Frente a lo anterior, aflora la siguiente pregunta: ¿la clase invertida puede, como metodología, aportar al desarrollo de habilidades de mayor desarrollo cognitivo? Lo primero que debemos plantear es que, definitivamente, este tipo de metodología, al ser parte de lo que denominamos metodologías activo-participativas, asegura no solo un rol activo del estudiante, sino, además, una vinculación con campos más avanzados del conocimiento que la sola memorización o la mera comprensión, ampliando el horizonte, $\mathrm{y}$, en directa respuesta a nuestro cuestionamiento, afirmar que el flipped classroom tiene vocación hacia el desarrollo de todo tipo de habilidades superiores en torno al aprendizaje, como lo señala Arráez y otros (2018: 156) cuando comentan que:

Se destacan: una mayor atención sostenida [...] el desarrollo de un pensamiento crítico, una mejora [...] sustancial tanto en la comunicación escrita como en las habilidades de razonamiento [...] y un incremento de la integración de los conocimientos [...] además esta metodología mejora la gestión del conocimiento del alumnado, ya que los materiales facilitados por el profesor les ayudan a preparar cada sesión de clase y, por tanto, les ayuda a mantener un papel más activo en el proceso de enseñanza-aprendizaje, el cual resulta clave para alcanzar una mayor tasa de retención en los contenidos. 


\section{La tecnología como herramienta natural del flipped classroom}

Si bien la clase invertida puede concebirse sin apoyo de tecnologías en contextos normales y presenciales, ello no es óbice para desechar la aplicación de tecnologías como apoyo y medio facilitador a la hora de implementar el método, ya que la tecnología puede constituirse en el mejor puente para el acercamiento y la comunicación entre estudiante y docente (Castellanos-Claramunt, 2020: 170).

La utilización de las tecnologías de la información y la comunicación permite avanzar hacia una nueva forma de llevar el proceso educativo, en la que se mejora el aprendizaje por parte de estudiante y la enseñanza por parte del docente, ampliando la práctica educativa a niveles consagrados y experimentales.

Las tecnologías de la información y la comunicación deben concebirse como lo que son, herramientas al servicio de la educación y no un sustituto del docente (Flores y otros, 2020: 4). Entenderlo en el segundo sentido presenta un doble riesgo. Por un lado, desviar la tarea del docente y permitirle que se refugie en la tecnología para suplirlo y, en segundo sentido, restarle la relevancia que detenta el docente en cualquier proceso educativo y bajo cualquier método o metodología educativa, sobre todo cuando es desde la visión del estudiante en que se prescinde de la función del profesor por la tecnología.

De esta forma, entender las tecnologías como una herramienta más, permite articular el proceso educativo a través de distintos insumos necesarios, sin relevar la importancia del docente en la tarea de educar, guiar y acompañar el proceso de aprendizaje de sus estudiantes. Así, la inclusión de las tecnologías de la información y la comunicación ha sido una práctica creciente en todo tipo de educación, como lo explican Flores y otros (2020: 7):

La inclusión de las tecnologías de la información y la comunicación en entornos educativos se ha convertido en una obligación, pero sobre todo en una necesidad para generar procesos de innovación, los cuales permitan elevar la calidad, la equidad, el acceso y la pertinencia en la educación superior [...] Pero también se convierte en un factor importante que atender en los niveles de educación media superior, en donde el estudiantado debe desarrollar y adquirir las competencias necesarias para su correcto desempeño, en sus estudios universitarios y laborales.

Frente a la ventaja que presenta la tecnología en la educación, es también relevante destacar que, frente a la creciente práctica de innovar por parte de docentes de todo tipo de educación, es que las tecnologías de la información y la comunicación se transforman en un importante aliado de este tipo de docente. En este sentido, podemos afirmar que la clase invertida se apoya de manera casi natural de la tecnología, como un requisito indispensable de la educación del siglo XXI (Jiménez y otros, 2019: 126). 


\section{Aplicación y resultados de flipped classroom en la clase de Introducción al Derecho Civil}

De acuerdo con lo reseñado en los apartados anteriores, el estado de necesidad de la educación superior en general planteó algunos desafíos importantes en torno a la continuidad del servicio educacional y, en forma puntual, a los docentes el requerimiento inmediato de decisiones para desarrollar de la mejor manera posible el proceso aprendizaje-enseñanza.

Es en este contexto en el que se nos plantearon diferentes inquietudes que debían ser abordadas de manera creativa, por un lado, y por otro de manera urgente, de modo de que las decisiones a tomar debieran, a su vez, tener un alto contenido centrado en el conocimiento, pero también asegurar el aprendizaje y la adquisición de competencias declaradas en los programas.

La decisión particular se centró en la elaboración de ciertos criterios de análisis asociados a los requerimientos que se debían cumplir, esto es, criterios asociados a un aprendizaje significativo o profundo, el desarrollo de competencias asociadas al nivel y la asignatura impartida, además de la retención de los estudiantes, si tenemos en consideración que la intervención didáctica se aplicó a un curso de primer año.

Como se anticipó, la búsqueda de respuestas llevó a plantearse una serie de acciones y metas a conseguir, por lo que surgieron los hitos a mantener o conseguir en el proceso educativo, al que denominamos como plan o desafío RAMPA, del acrónimo RA (rendimiento académico), en el que la meta a conseguir implicó no solo mantener o incrementar el desempeño individual de cada estudiante, sino su influencia total, sea en el resto de grupo o al interior del aula (Navarro, 2003: 3). Por esto, el rendimiento considera la calificación obtenida de una evaluación solo como una parte del rendimiento, ya que la participación en clase y la calidad de esa participación forman, en su conjunto, el hito rendimiento académico. Los criterios específicos para este hito apuntan a la: i) aplicación de la teoría a situaciones prácticas a través del estudio de casos; ii) aprendizaje profundo los contenidos; iii) aumento de rendimiento académico en general; y iv) desarrollo de habilidades de orden superior.

La M (motivación), tiene en consideración que los estudiantes de primer año, en contextos de normalidad, pueden perder el interés por la carrera que comienzan a estudiar, por un sinnúmero de variables a las que se le sumaba, en esta oportunidad, la modalidad virtual, que fue, sin lugar a duda, un obstáculo para los estudiantes en general. Esta cuestión fue particularmente observada en nuestros estudiantes de primer año, para quienes los primeros pasos en el derecho fueron recorridos con incertidumbre de su aprendizaje y, muchas veces, con un escaso entendimiento de lo que estaban estudiando, sin desconocer la calidad de novatos en la cultura académica universitaria (Poblete y otros, 2020: 25). Los criterios específicos apuntaron: i) al aumento del interés por aprender derecho; ii) al entendimiento de la utilidad del apren- 
dizaje para la formación profesional; y iii) a que el proceso de aprendizaje-enseñanza fuera entretenido o didáctico.

La letra P (participación activa del estudiante) busca directamente el aprendizaje activo, en el que el alumno se implica, se esfuerza y se compromete con su aprendizaje (Miguel y otros, 2012: 81), no solo estudiando para una prueba o para evaluación calificada, sino que en todo el proceso, desde la visualización del video tutorial, la lectura previa de un documento, la elaboración de actividades, sea esta individual o grupal, y la participación en clases a través de preguntas, respuestas o cualquier otro tipo de intervención. Los criterios de mayor especificidad estuvieron en torno a: i) el trabajo de manera efectiva con el equipo; ii) la posibilidad de expresar y compartir respuestas a los casos planteados; y iii) la oportunidad de interactuar con el docente.

Finalmente, la A (aprovechamiento efectivo del tiempo de clases) comprende el tiempo fuera del aula como el tiempo personal del estudiante en actividades dirigidas y planificadas por el docente, en las que el estudiante se prepara para la clase, como aquel tiempo dentro de la clase que comprende el tiempo instruccional y el tiempo de interacción entre los estudiantes, y entre los estudiantes y el docente (Martinic, 2015: 492). Estos criterios específicos se centraron en: i) adquirir en las clases un aprendizaje de calidad; ii) disponer de la oportunidad para plantear dudas; y iii) reflexionar y organizar ideas para desarrollar las actividades en el aula.

A partir de los criterios de análisis fijados, el objetivo central fue asegurar, por una parte, a través de un escenario completamente virtual, la calidad de la enseñanza y, por otro, la calidad de los aprendizajes necesarios en la formación del estudiante de primer año de la carrera de Derecho. Esto, a través de la intervención didáctica «clase invertida» o flipped classroom, de la que se dará cuenta en las siguientes líneas a través de la experiencia aplicada en la asignatura de Introducción al Derecho Civil. Además, se mostrarán los resultados obtenidos a partir de la apreciación y del grado de satisfacción de los estudiantes asistentes al curso.

\section{Diseño y desarrollo de la intervención didáctica}

\section{Descripción del contexto y de los participantes}

La experiencia que se expone en este trabajo se desarrolló al interior de la Facultad de Derecho de la Universidad Santo Tomás, específicamente en la Escuela de Derecho de Antofagasta, en la asignatura de Introducción al Derecho Civil, correspondiente al segundo semestre de primer año de la cohorte de 2020.

La asignatura en cuestión se estructura a partir de tres unidades de aprendizaje: La unidad I, denominada Código Civil chileno, con una carga de 42 horas pedagógicas; la unidad II, llamada Teoría de la Ley, con una carga de 30 horas pedagógicas; y la unidad III, denominada Sujetos de Derecho, con 30 horas pedagógicas. 
Por lo tanto, el semestre se estructuró de la siguiente manera: la unidad I se impartió a través de un sistema tradicional, es decir, una clase tradicional, con la entrega del material después de desarrollados los contenidos por el docente y sin actividades o evaluaciones formativas. Esta primera unidad fue evaluada únicamente a través de una prueba denominada solemne, calificada y de carácter sumativa.

Por otro lado, las unidades II y III fueron trabajadas con los estudiantes bajo el método de clase invertida o flipped classroom, práctica que será descrita más adelante.

Frente a la decisión de estructurar las metodologías de la manera en que se ha presentado, cabe señalar que una de las premisas a manejar era la posibilidad de que los estudiantes pudieran contrastar, en el mismo semestre, en la misma asignatura y con el mismo docente, la aplicación y, a su vez, la diferenciación de las prácticas presentadas, con énfasis en la evaluación del método con el que se estaba interviniendo, como es la clase invertida.

En relación con el número de participantes en la experiencia, la matrícula durante el semestre fue de 36 estudiantes, que es el número al que se sometió la intervención didáctica, las evaluaciones, tanto formativas como sumativas, además de la encuesta final de satisfacción.

\section{Desarrollo de la propuesta metodológica}

La experiencia en general tuvo aproximadamente un desarrollo temporal de 17 semanas, en las primeras 7 se desarrolló la unidad I bajo el método tradicional de clases y una evaluación sumativa al final de la unidad, mientras que en las restantes 10 semanas se desarrollaron las unidades II y III a través del método de clase invertida, consignándose una evaluación sumativa final, dos evaluaciones formativas, además de las actividades o tareas semanales. Para el desarrollo del método de clase invertida, la intervención se organizó de la siguiente forma.

La primera etapa consistió en que, al inicio del semestre, se les informó a todos los estudiantes del curso de Introducción al Derecho Civil que, durante el semestre, se aplicarían las dos metodologías señaladas, especificando las unidades, los tiempos y los métodos a aplicar, con especial énfasis en la descripción de lo que es la clase invertida y su aplicación en el aula.

En la segunda etapa tuvo lugar la elaboración y la preparación de los recursos para trabajar el método de clase invertida. Si bien la plataforma tecnológica ha sido una herramienta a disposición del proceso educativo a través del aula virtual desde hace años, lo cierto es que durante 2020 su utilización fue masiva, por lo que el escenario donde se alojaría el material era altamente cotidiano y conocido por los estudiantes. Sin embargo, la organización de los materiales y su temporalidad fueron diferentes en la aplicación de la clase invertida. Es por ello que, dentro de las actividades previas por parte del docente, estuvo la preparación de las unidades en la plataforma virtual. 
Además, se debe resaltar que, en esta etapa, el docente debe elaborar el material de manera consiente, encausada y coordinada con los contenidos y las habilidades a desarrollar. Entre los materiales elaborados están la selección de textos de lectura, la elaboración de cápsulas o videos y las actividades prácticas que debían desarrollar los estudiantes.

La tercera etapa consistió en la aplicación del método de clase invertida. Para ello, se destacan las siguientes acciones realizadas: i) descripción del método a los estudiantes, explicación del rol activo que se espera de ellos, explicación de los pasos a seguir y de las tareas a realizar antes de cada clase; ii) se cargan los primeros recursos junto con las instrucciones directas a realizar, esto sucede cada vez que termina la última clase y se anticipan las acciones a seguir de manera de asegurar que, en la clase siguiente, los estudiantes lleguen con el texto leído, el video visualizado o la actividad realizada. En esta etapa se activa la participación de los estudiantes, de manera de asegurar el contenido previo, básico y mínimo.

En la cuarta etapa se debe complementar el método de clase invertida con la clase tradicional. Es por eso que, en aquellas clases en las que se aplique en su totalidad el flipped classroom, el docente debe enfocar la clase en la participación activa de los estudiantes, a través de la revisión de las actividades realizadas en su hogar, la retroalimentación y el espacio para preguntas o el debate de ideas. Puede ocurrir también que la clase se trasforme en el espacio para elaborar y desarrollar la actividad práctica, dejando la corrección y el espacio de la interacción para la próxima sesión. Es importante reconocer que las actividades las pueden realizar los estudiantes, ya sea en clase o en el aula, dependiendo de donde las proyecte el docente, lo que no obsta a que el profesor sea quien deba decidirlo a través de criterios técnicos, de modo que disponga de tareas para el hogar que sean fáciles de resolver y no presenten algún grado de dificultad para el estudiante y sea el pretexto para no hacerla o, simplemente, sea causa de desmotivación, por lo que se recomienda que las actividades prácticas de mayor dificultad, así como los contenidos teóricos del mismo grado, sean desarrollados en clase por el docente.

La quinta etapa corresponde a la evaluación en general. Primero, la evaluación formativa a través de las actividades prácticas. Acto seguido, y como es el caso, lo aprendido y practicado fue evaluado a través de una prueba solemne sumativa. En este sentido, la evaluación debe disponer de diversos ítems que aseguren variedad, pero, por sobre todo, que combinen las diferentes habilidades desarrolladas, y no solo el conocimiento o la memorización, mucho menos en los contextos virtuales en los que el riesgo de copia es altamente probable.

La sexta etapa es la de la evaluación de la experiencia, ya sea desde la óptica de los resultados académicos plasmados en la evaluación sumativa como desde la evaluación general que realizan los estudiantes del método de clase invertida, que en 
nuestro caso fue a través de una encuesta de satisfacción, lo que permitió, sin duda, recoger algunos datos que serán expuestos en los párrafos siguientes.

\section{Evaluación de la intervención didáctica clase invertida}

La experiencia descrita hasta este apartado hemos querido evaluarla a partir de la propia vivencia de los estudiantes, en especial, bajo los criterios que en su momento fueron socializados, los que forman parte esencial de la intervención.

\section{Instrumento}

La encuesta de satisfacción aplicada a los estudiantes consistió en un set de preguntas utilizando una Escala de Likert, en la que 1 correspondía a «muy en desacuerdo»; 2 a «en desacuerdo»; 3 a «ni de acuerdo ni en desacuerdo (neutro)»; 4 a «de acuerdo»; y 5 a «muy de acuerdo», mientras que el procedimiento de aplicación y la recogida de datos fue virtual a través de la plataforma Google Forms.

Las 13 preguntas del cuestionario se organizaron y fueron diseñadas a partir del desafío RAMPA, ya descrito, y de los hitos que lo componen, en un contexto del método de clase invertida.

De esta forma, la evaluación permitió evaluar, a través de la satisfacción del estudiantado: i) el grado de satisfacción de la intervención metodológica, es decir, directamente el método de aula invertida; y ii) a nosotros en particular, lo que nos permitió evaluar el cumplimiento de los hitos propuestos a través de la clase invertida. En la tabla 1 se puede visualizar la estructura de las preguntas realizadas.

\section{Resultados}

De los 36 estudiantes matriculados, 34 de ellos dieron respuesta a la encuesta de satisfacción, lo que representa el 94,4\% del grupo total que fue sometido a la intervención didáctica. Los datos extraídos fueron los siguientes y se pueden apreciar tanto en la tabla 2 como en la figura 1.

\section{Rendimiento académico}

De acuerdo con el hito de rendimiento académico, es posible afirmar que, desde la perspectiva de los estudiantes, el aprendizaje profundo de los contenidos (R2) se logró con el 79,3\%; el aumento de rendimiento académico ( $\mathrm{R}_{3}$ ) se logró con el 82,3\%; la aplicación de la teoría a situaciones prácticas (R1) también alcanza un alto grado de aceptación con el 82,3\%, mientras que el desarrollo de competencias superiores (R4) alcanzó también el 82,3\%. Los datos mencionados se entienden considerando todas las respuestas afirmativas, de acuerdo y muy de acuerdo. Por todo esto, el rendimien- 
Tabla 1. Elaboración autoadaptada de Moya y Soler (2019)

\begin{tabular}{|l|l|}
\hline Criterios de análisis & Preguntas \\
\hline RA (rendimiento académico) & $\begin{array}{l}\text { R.1 Aplicar la teoría a situaciones prácticas a través del estudio } \\
\text { de casos }\end{array}$ \\
\hline R.2 Aprender de manera profunda los contenidos \\
\hline R.3 Aumentar mi rendimiento académico
\end{tabular}

to académico en general presenta una alta valoración por parte del estudiantado, verificado por el aumento de sus calificaciones por un lado, pero, a su vez, por el desempeño que ellos mismos evalúan en sus procesos de autoaprendizaje, siendo el promedio general de todo el hito un $81,5 \%$.

Lo anterior se puede ratificar en la comparación del rendimiento a través de las calificaciones en las dos evaluaciones solemnes del semestre, recordando que el solemne 1 se evaluó a partir de los aprendizajes adquiridos bajo el modelo de clases tradicional, mientras que el solemne 2 evaluó aprendizajes bajo el método de clase invertida. Todo esto se puede observar en la tabla 3.

Un somero análisis permite diferenciar el rendimiento académico en su dimensión evaluación, en especial en el porcentaje de aprobación, esto es, nota 4,o hacia arriba, que aumenta del solemne 1 al solemne 2 de un $76,4 \%$ al 91,1\%, mientras que es posible observar, a su vez, que el grupo, en su mayoría, se desplaza desde el 4,o y 4,9 a 5,0 y 5,9, incrementando no solo el rendimiento de algunos reprobados, sino que del grupo en general, cuestión que también se puede observar en los de mayor rendi- 


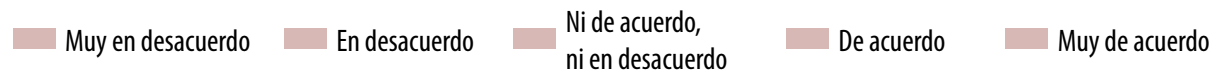

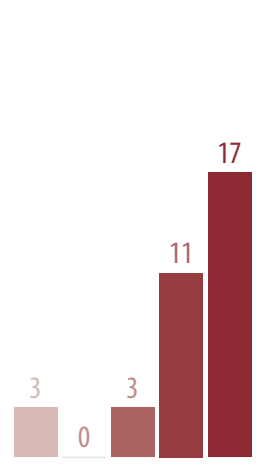

R1

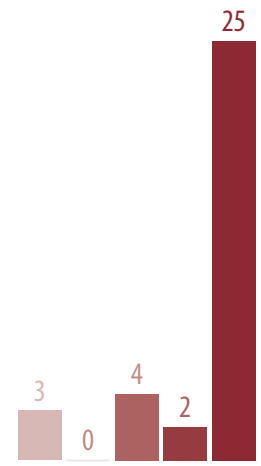

R2

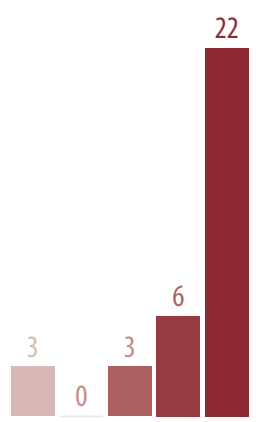

R3

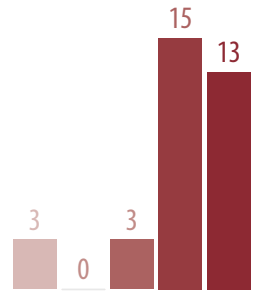

R4

Figura 1. Rendimiento.

Fuente: Encuesta de satisfacción en Google Forms.

Tabla 2. Rendimiento

\begin{tabular}{|c|c|c|c|c|c|c|c|c|c|c|}
\hline \multirow[t]{2}{*}{ Preguntas } & \multicolumn{2}{|c|}{ Muy en desacuerdo } & \multirow{2}{*}{$\begin{array}{c}\text { En desacuerdo } \\
\text { N }\end{array}$} & \multicolumn{3}{|c|}{$\begin{array}{l}\text { Ni de acuerdo, } \\
\text { ni en desacuerdo }\end{array}$} & \multirow{2}{*}{$\begin{array}{c}\text { De acuerdo } \\
\text { N }\end{array}$} & \multicolumn{3}{|c|}{ Muy de acuerdo } \\
\hline & N & $\%$ & & $\%$ & N & $\%$ & & $\%$ & N & $\%$ \\
\hline R1 & 3 & 8,8 & 0 & 0 & 3 & 8,8 & 11 & 32,3 & 17 & 50 \\
\hline R2 & 3 & 8,8 & 0 & 0 & 4 & 11,7 & 2 & 5,8 & 25 & 73,5 \\
\hline R3 & 3 & 8,8 & 0 & 0 & 3 & 8,8 & 6 & 17,6 & 22 & 64,7 \\
\hline R4 & 3 & 8,8 & 0 & 0 & 3 & 8,8 & 15 & 44,1 & 13 & 38,2 \\
\hline
\end{tabular}

Tabla 3. Rendimiento académico

\begin{tabular}{|lcccr|} 
& Prueba solemne 1 & \multicolumn{4}{c}{ Prueba solemne 2 } \\
& N & $\%$ & N & $\%$ \\
\hline Reprobados & 8 & 23,5 & 3 & 8,8 \\
Aprobados & 26 & 76,4 & 31 & 91,1 \\
\hline $1,0-3,9$ & 8 & 23,5 & 3 & 8,8 \\
$4,0-4,9$ & 19 & 55,8 & 8 & 23,5 \\
\hline $5,0-5,9$ & 6 & 17,6 & 17 & 50 \\
$6,0-7,0$ & 1 & 2,9 & 6 & 17,6 \\
\hline Total alumnos & 34 & & 34 & \\
\hline
\end{tabular}


miento. Si los resultados fueran graficados en una curva de Gauss, podríamos decir que el método de clase invertida desplazó completamente la curva hacia la derecha del gráfico, evidenciando el aumento del rendimiento de todo el grupo en general.

\section{Motivación}

La evaluación por parte de los estudiantes encuestados en el hito motivación (tabla 4 y figura 2) presenta un alto grado de satisfacción, ya que el promedio general de aceptación es del $85,2 \%$, el cual se puede desglosar de la siguiente forma: el aumento del interés por aprender (M1) se manifestó en una aceptación del 88,1\% de los encuestados; mientras que el reconocimiento de la utilidad del aprendizaje, en directa relación con la formación profesional del estudiante (M2), ponderó el 82,3\%. Finalmente, lo didáctico del proceso $(\mathrm{M})$ de traduce en el $85,2 \%$. De esta manera, se interpreta que el proceso aprendizaje-enseñanza se presenta como interesante, necesario, pertinente y propio por parte de la mayoría de los estudiantes. Resultan particularmente interesantes estos resultados, considerando que los estudiantes pertenecen a un primer año, bajo una modalidad virtual y con una materia compleja como es el derecho civil.

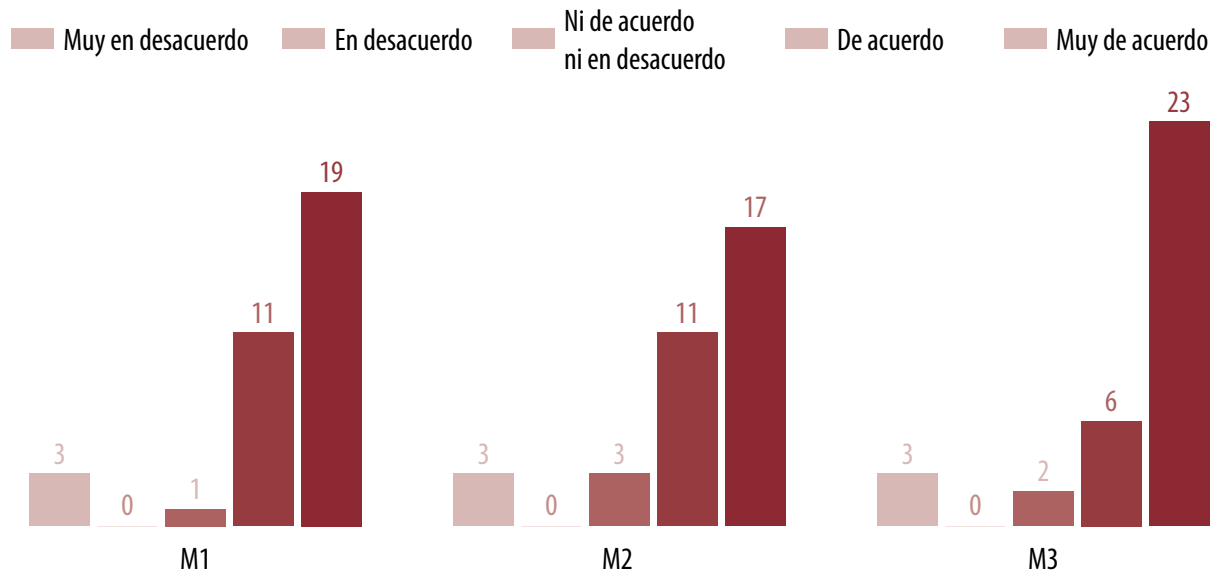

Figura 2. Motivación.

Fuente: Encuesta de satisfacción en Google Forms.

Tabla 4. Motivación

\begin{tabular}{|lrrrrrrrrrrr} 
Preguntas & Muy en desacuerdo & En desacuerdo & \multicolumn{3}{c|}{$\begin{array}{c}\text { Ni de acuerdo } \\
\text { ni en desacuerdo }\end{array}$} & \multicolumn{3}{c|}{ De acuerdo } & \multicolumn{4}{c}{ Muy de acuerdo } \\
& N & $\%$ & N & $\%$ & N & $\%$ & N & $\%$ & N & $\%$ \\
\hline M1 & 3 & 8,8 & 0 & 0 & 1 & 2,9 & 11 & 32,3 & 19 & 55,8 \\
M2 & 3 & 8,8 & 0 & 0 & 3 & 8,8 & 11 & 32,3 & 17 & 50 \\
M3 & 3 & 8,8 & 0 & 0 & 2 & 5,8 & 6 & 17,6 & 23 & 67,6 \\
\hline
\end{tabular}




\section{Participación}

En cuanto a la participación activa de los estudiantes, los resultados no difieren de los que se han analizado hasta ahora en los otros hitos (tabla 5 y figura 3). En términos generales, el nivel de satisfacción del hito es del 82,2 \%, mientras que el trabajo efectivo con el equipo ( $\mathrm{P} 1$ ) alcanzó el $88,1 \%$ y la posibilidad de participar e interactuar a través de presentación de respuestas o resolución de casos $\left(\mathrm{P}_{2}\right)$ presentó una cifra similar, siendo la posibilidad de interactuar con el docente el ítem disminuido con el 70,5\%, que si bien se puede considerar aceptable, al interior del hito es el de menor satisfacción. Lo anterior podría explicarse de acuerdo con lo observado por el escenario virtual que dificulta, en todo sentido, la comunicación y la interacción en grupos grandes de personas, lo que genera, sin duda, barreras autoimpuestas por los propios estudiantes. En términos generales, es posible observar que el método de clase invertida no solo permite la participación activa del estudiante en su proceso de aprendizaje, sino que, además, la fomenta, ya sea gestionando sus tareas o participando activamente del desarrollo de la clase y la retroalimentación de las actividades.

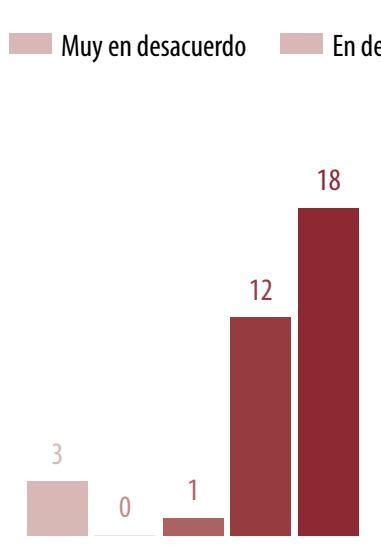

P1

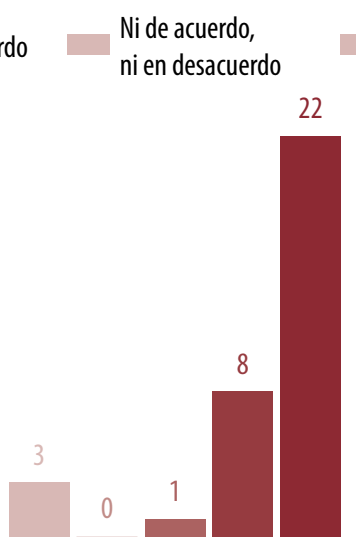

P2
De acuerdo

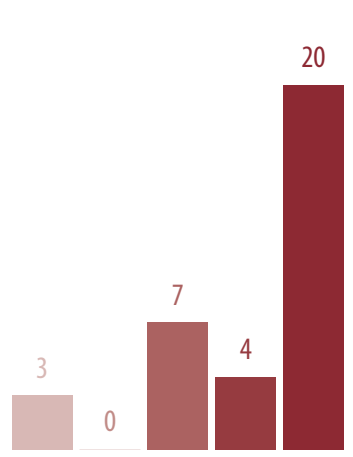

P3

Figura 3. Participación.

Fuente: Encuesta de satisfacción en Google Forms.

Tabla 5. Participación

\begin{tabular}{|lrrrrrrrrrrr|r|} 
Preguntas & Muy en desacuerdo & En desacuerdo & \multicolumn{3}{c|}{$\begin{array}{c}\text { Ni de acuerdo, } \\
\text { ni en desacuerdo }\end{array}$} & \multicolumn{3}{c}{ De acuerdo } & \multicolumn{4}{c}{ Muy de acuerdo } \\
& N & $\%$ & N & $\%$ & N & $\%$ & N & $\%$ & N & $\%$ \\
\hline P1 & 3 & 8,8 & 0 & 0 & 1 & 2,9 & 12 & 35,2 & 18 & 52,9 \\
P2 & 3 & 8,8 & 0 & 0 & 1 & 2,9 & 8 & 23,5 & 22 & 64,7 \\
P3 & 3 & 8,8 & 0 & 0 & 7 & 20,5 & 4 & 11,7 & 20 & 58,8 \\
\hline
\end{tabular}




\section{Aprovechamiento de la clase}

El hito aprovechamiento efectivo del tiempo de clases es también valorado favorablemente por los estudiantes (tabla 6 y figura 4) con el 84,2\% en términos generales. Con el $82,3 \%$, está el convencimiento de que en la clase adquieren un aprendizaje de calidad (A1), por lo que la clase es el escenario más propicio para ello. Por otro lado, el tiempo para solicitar ayuda, corrección o respuestas del docente (A2) alcanza el $82,3 \%$, mientras que la posibilidad de reflexionar y organizar ideas para desarrollar las actividades en clases alcanza el 88,2\%. Lo anterior refleja la lógica y la mecánica que persigue la clase invertida, la distribución de los tiempos de trabajo antes de llegar a la clase de aquellos que se articulan y desarrollan en la clase, con la posibilidad de que la explicación del profesor en el aula sea de aquellos contenidos de mayor dificultad, la realización de actividades que requieran el apoyo y la guía presencial del docente, y los tiempos necesarios para preguntar, reflexionar y debatir.

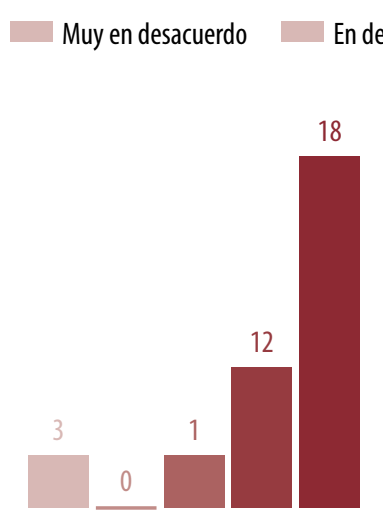

A1
En desacuerdo

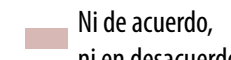
ni en desacuerdo
De acuerdo

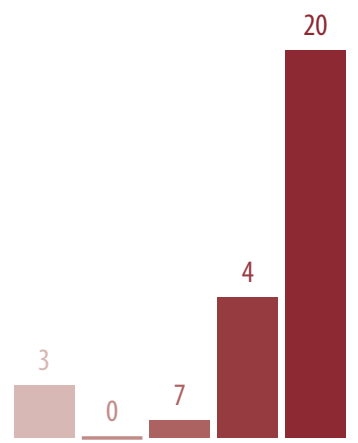

A3

Figura 4. Aprovechamiento de la clase.

Fuente: Encuesta de satisfacción en Google Forms.

Tabla 6. Aprovechamiento de la clase

\begin{tabular}{|c|c|c|c|c|c|c|c|c|c|c|}
\hline \multirow[t]{2}{*}{ Preguntas } & \multicolumn{2}{|c|}{ Muy en desacuerdo } & \multirow{2}{*}{$\begin{array}{c}\text { En desacuerdo } \\
\mathrm{N}\end{array}$} & \multicolumn{3}{|c|}{$\begin{array}{l}\text { Ni de acuerdo, } \\
\text { ni en desacuerdo }\end{array}$} & \multirow{2}{*}{$\begin{array}{c}\text { De acuerdo } \\
\text { N }\end{array}$} & \multicolumn{3}{|c|}{ Muy de acuerdo } \\
\hline & N & $\%$ & & $\%$ & N & $\%$ & & $\%$ & N & $\%$ \\
\hline $\mathrm{A} 1$ & 3 & 8,8 & 0 & - & 3 & 8,8 & 8 & 23,5 & 20 & 58,8 \\
\hline$A 2$ & 3 & 8,8 & 0 & - & 3 & 8,8 & 9 & 26,4 & 19 & 55,9 \\
\hline A3 & 3 & 8,8 & 0 & - & 1 & 2,9 & 8 & 23,5 & 22 & 64,7 \\
\hline
\end{tabular}




\section{Consideraciones finales}

La emergencia sanitaria que cubrió la realidad mundial y, en especial, la educación, obligó a modificar una serie de prácticas tradicionales, incorporando nuevas metodologías o adaptando las existentes a las diferentes realidades y necesidades. En tanto, se ha configurado un escenario nuevo y virtual, el que necesariamente, creemos, ha llegado para quedarse en sus diversas aplicaciones.

La clase invertida forma parte del gran abanico de las denominadas metodologías activa-participativas. Es por ello que debemos considerarla como una de las tantas alternativas que se pueden adoptar en el aula de clases, de manera de hacerla más efectiva, aumentar el rendimiento académico de los estudiantes y alcanzar los objetivos planteados de una manera distinta a la tradicional. La amplitud y la flexibilidad del método le permite ser aplicada y complementada desde un contenido específico a una unidad temática o a un semestre académico completo, sin limitación alguna. La decisión de aplicación, el tiempo y los fines que se persiguen se presenta como una tarea necesaria del docente, en el que deberá evaluar su aplicación y lo que necesariamente pretende conseguir al invertir la clase.

La experiencia permite verificar que la adopción de la metodología de clase invertida es un proceso complejo en su inicio, ya que debe movilizarse a los estudiantes, sacarlos del rol pasivo e incorporarlos en un rol activo. Esto puede llevar días o incluso semanas. Eventualmente, se podría dar la situación de estudiantes que definitivamente no se suman al proceso, cuestión que es posible observar en nuestro caso, en el que el $8,8 \%$ de los encuestados no está de acuerdo con la clase invertida, evidenciado en todos los hitos y todas las preguntas. Lo anterior, constituye un riesgo cuando el número de estudiantes no se adecúa al método de clase invertida y, por tanto, la eficacia de él falla. Sin duda, esto lo asumimos como responsabilidad y debilidad en tanto gestores de la experiencia y como docentes. Nos referimos al $8,8 \%$ no vinculado o comprometido con la intervención didáctica.

La intervención didáctica presentada cumple con todo lo propuesto en el desafío RAMPA. Así, nos permitió controlar los criterios de análisis que se presentaban al inicio de segundo semestre como problemáticos o desafiantes y lograr con ello la identificación de esas falencias, no solo por parte del docente, que es el que las identifica inicialmente, sino que el conocimiento e interiorización por parte de los estudiantes, quienes, a partir de sus debilidades, desventajas o problemas, se incorporan al proceso como ejecutores de la solución.

Es necesario establecer que, si bien el instrumento no presenta una validación, o la muestra corresponde a una población pequeña, los resultados obtenidos en la encuesta de satisfacción permiten concluir que a los estudiantes les parece un buen método para aprender y para su formación, ya que incrementa la motivación y la disposición para aprender, junto con el rendimiento académico y la participación 
de ellos en todos los momentos del proceso. Lo anterior no debe interpretarse en relación con la clase tradicional, ya que la experiencia y la encuesta no tienen un fin comparativo, ni mucho menos ponderar la calidad de los métodos y su eficacia en el aula. Como se planteó en líneas anteriores, consideramos que la clase tradicional y la clase a partir de una metodología activo-participativa debe, necesariamente, complementarse y subsidiarse para la obtención de resultados de aprendizajes esperados.

Si bien la encuesta de satisfacción nos brinda información relevante del desarrollo del semestre por parte de los estudiantes, lo cierto es que, a través de la propia experiencia, pudimos observar cómo otros criterios no evaluados se fueron desarrollando en los participantes. El pensamiento crítico, la reflexión y el razonamiento jurídico se fueron visualizando en las diversas actividades desarrolladas, en especial aquellas que planteaban problemas prácticos y cuya solución debían construir a partir de la ley, la teoría, la creatividad y el pensamiento divergente.

El trabajo colaborativo fue otro punto observado, destacado y bien evaluado, ya que gran parte del trabajo que debieron realizar fue de manera grupal, con un alto grado de responsabilidad en la planificación y resolución de los problemas, destacándose en algunos grupos la búsqueda de información más allá de lo necesario, resaltando la curiosidad con la que trabajaron muchos de los estudiantes.

\section{Referencias}

AваD, Emilio у Mariana González (2019). «Análisis de las competencias en la educación superior a través de flipped classroom». Revista Iberoamericana de Educación, 80 (2): 29-45. DOI: 10.35362/rie8023407.

AbeyseKera, Lakmal y Phillip Dawson (2015). «Motivation and cognitive load in the flipped classroom: Definition, rationale and a call for research». Higher Education Research \& Development, 34 (1): 1-14. DOI: 10.1080/07294360.2014.934336.

Aedo, Cristian (2014). «Formación por competencias y la enseñanza del derecho: Universidad de Chile». Revista Pedagogía Universitaria y Didáctica del Derecho, (1) 1: 104-113. DOI: 10.5354/0719-5885.2015.35966.

Albornoz, Jocelyn, José Maldonado, Cristian Vidal y Erika Madariaga (2020). «Impacto y recomendaciones de clase invertida en el proceso de enseñanzaaprendizaje de geometría». Formación universitaria, 13 (3): 3-10. DOI: 10.4067/ So718-50062020000300003.

AlCántara, Armando (2020). «Educación superior y covid-19: Una perspectiva comparada». En Educación y pandemia: Una visión académica (pp. 75-82). Centro Cultural Universitario, UNAM, Ciudad de México. Disponible en https://bit. ly/3mCSLPR.

ANDRADE, Ender y Edixon Chacón (2018). «Implicaciones teóricas y procedimentales de la clase invertida». Pulso, 41: 251-267. Disponible en https://bit.ly/2Mc5Xvd. 
Arráez, Graciela, Alejandro Lorenzo, Marcos Gómez y Gonzalo Lorenzo (2018). «La clase invertida en la educación superior: Percepciones del alumnado». International Journal of Developmental and Educational Psychology, 2 (1): 155-162. Disponible en https://bit.ly/32FagfI.

Bartolomé-Pina, Antonio (2020). «Cambios educativos en tiempos de pandemia». Innovaciones Educativas, 22: 13-16. DOI: https://bit.ly/344yJas.

BENFELD, Johann (2016). «La discusión sobre enseñanza del derecho en Chile dentro del nuevo paradigma universitario: Una tarea pendiente». Revista de Derecho, 23 (1): 143-171. Disponible en https://bit.ly/3qoCr64.

Berenguer, Cristina (2016). «Acerca de la utilidad del aula invertida o flipped classroom». XIV Jornadas de Redes de Investigación en Docencia Universitaria (pp. 1.466-1.480). Instituto de Ciencias de la Educación, Universidad de Alicante.

Bergmann, Jonathan y Aaron Sams (2014). Dale la vuelta a tu clase. Madrid: SM.

-. (2017) «Flipped learning: Gateway to student engagement». Center for Educational Policy Studies Journal, 7 (3): 173-176.

Carranza, Priscilla y Geraldine Zamora (2020). «Desafíos y oportunidades en tiempos del covid-19: Contexto pedagógico desde la Universidad Nacional y la Universidad Estatal a Distancia». Innovaciones Educativas, 22. DOI: 10.22458/ ie.v22iespecial.3154.

Castellanos-Claramunt, Jorge (2020). «Innovación docente y TIC desde la perspectiva de la docencia en derecho». Revista Pedagogía Universitaria y Didáctica del Derecho, 7 (2): 167-184. DOI: 10.5354/0719-5885.2020.57150.

Cepal y Unesco. (2020). La educación en tiempos de la pandemia de covid-19. Disponible en https://bit.ly/3pyW5gP.

Cobeña, Miguel y María Rodríguez (2019). «La clase invertida como modelo de investigación pedagógica». Cienciamatria, 5 (1): 3-15. DOI: 10.35381/cm.v5i1.236.

Coloma, Rodrigo (2006). "Hacia una enseñanza del derecho efectiva». Revista Escuela de Derecho, 7 (7): 123-141.

Elgueta, María Francisca y Eric Palma (2015). «De la enseñanza del derecho a la didáctica general del derecho: Instituto de Investigación Jurídica». Universidad Autónoma de México. Disponible en https://bit.ly/3EM9Vkl.

FigueroA, Mauricio (2020). "El aprendizaje basado en investigación como alternativa didáctica del proceso de aprendizaje-enseñanza en el derecho". Revista Pedagogía Universitaria y Didáctica del Derecho, 7(1), 237-259. DOI: 10.5354/07195885.2020.54858.

Flores, Linda, María Veytia y Javier Moreno (2020). «Clase invertida para el desarrollo de la competencia: Uso de la tecnología en estudiantes de preparatoria». Revista Educación, 44 (1). Disponible en https://bit.ly/32FEmv9.

Galindo, Héctor y María-José Bezanilla (2019). «Una revisión sistemática de la metodología flipped classroom a nivel universitario en España». Innoeduca. In- 
ternational Journal of Technology and Educational Innovation, 5 (1): 81-90. DOI: 10.24310/innoeduca.2019.v5i1.4470.

Gámiz, Vanesa y María Jesús Gallego. (2016). "Modelo de análisis de metodologías didácticas semipresenciales en Educación Superior". Educación XX1, 19(1), 39-61. DOI: 10.5944/educXX1.13946.

Gómez, Carmen y Francisco López. (2019). «Application of innovative teachinglearning methodologies in the classroom. Coaching, flipped-classroom and gamification. A case study of success». Multidisciplinary Journal for Education, Social and Technological Sciences, 6 (1): 46-70. Disponible en https://bit.ly/3px7jlK.

GonzÁlez, Carmen, Isabel Feito, Marcos González, Laura Valdunciel, José Luis Álvarez y Nicolás Sarmiento Alonso (2017). «Modelo de enseñanza-aprendizaje basado en la clase invertida: Una experiencia de innovación docente en diferentes niveles educativos». Revista Infancia, Educación y Aprendizaje, 3 (2): 59-65. DOI: 10.22370/ieya.2017.3.2.700.

Hinojo, Francisco, Inmaculada Aznar, José Romero y José Marín (2019). «Influencia del aula invertida en el rendimiento académico: Una revisión sistemática». Campus Virtuales, 8 (1): 9-18.

JimÉNEZ, Amparo y Francisco Robles (2016). «Las estrategias didácticas y su papel en el desarrollo del proceso de enseñanza aprendizaje». Educaticonciencia, 9 (10): 106-113. Disponible en https://bit.ly/3zz $S_{3 j} B$.

JimÉnez, David, Patricia Sancho y Sergio Sánchez (2019). «Perfil del futuro docente: Nuevos retos en el marco del EEES». Contextos Educativos, o (23): 125-139. DOI: 10.18172/con.3471.

Lıma, Hélder, Aline Battisti, Fernanda Bordignon, Fernanda Torres, Gabriel Gomes, Marcelo Henrique Oliveira, Mariana Gomide, Nádia Kienen, Otávio Beltramello, y, Valquiria Maria Gonçalves (2020). «Ensino superior em tempos de pandemia: Diretrizes a gestao universitaria». Educação \& Sociedade, 41: 1-21. DOI: 10.1590/ es.238957.

Lazo, Patricio (2011). «Formación jurídica, competencias y métodos de enseñanza: Premisas». Ius et Praxis, 17 (1): 249-262.

MARTín, Déborah y Raúl Santiago (2016). «Flipped Learning en la formación del profesorado de secundaria y bachillerato: Formación para el cambio». Contextos Educativos, 2016: 117-134. DOI: 10.18172/con.2854.

MARTINIC, Sergio (2015). «El tiempo y el aprendizaje escolar la experiencia de la extensión de la jornada escolar en Chile». Revista Brasileira de Educação, 20 (61): 479-499. DOI: 10.1590/S1413-24782015206110.

Melo, Lina y Ramiro Sánchez (2017). «Análisis de la percepción de los alumnos sobre la metodología flipped classroom para la enseñanza de técnicas avanzadas en laboratorios de análisis de residuos de medicamentos veterinarios y contaminantes». Educación Química, 28 (1): 30-37. DOI: 10.1016/j.eq.2016.09.010. 
Miguel, José, David López y Miryam Martin (2012) «¿Una participación activa del alumno pronostica una buena nota en el examen?». Working Papers on Operations Management, 3 (2): 71-83. DOI: 10.4995/wpom.v3i2.1097.

Mok, Heeng (2014). «Teaching tip: The flipped classroom». Journal of Information Systems Education, 25 (1): 7-11.

MonTes, Ana, Víctor Villalobos y Warner Ruiz (2020). «Estrategias didácticas empleadas desde la presencialidad remota en la división de educación para el trabajo de la Universidad Nacional en tiempos de pandemia». Innovaciones Educativas, 22: 243 -262. DOI: 10.22458/ie.v22iEspecial.3251.

Moya, María y Carolina Soler (2019). «La clase invertida o flipped classroom en la enseñanza de materias jurídicas. En Rosabel Roig-Vila (editora), Investigación e innovación en la enseñanza superior: Nuevos contextos, nuevas ideas (pp. 1.2261.235). Barcelona: Octaedro.

Navarro, Rubén (2016). «El rendimiento académico: Concepto, investigación y desarrollo». REICE, 1 (2): 1-16. Disponible en https://bit.ly/3pwOpLJ.

Perdomo, William (2016). «Estudio de evidencias de aprendizaje significativo en un aula bajo el modelo flipped classroom». Edutec: Revista Electrónica De Tecnología Educativa, 55. DOI: 10.21556/edutec.2016.55.618.

PÉrez, Francisco (2017). «La metodología suite desde la metodología flipped classroom: Una propuesta para la asignatura de Análisis». Consonancias, 1: 84-114.

Poblete, Claudia, Lisbeth Arenas, y Alejandro Córdova (2020). «Alfabetización académica en derecho: Experiencia y resultados de un programa de escritura». Revista Pedagogía Universitaria y Didáctica del Derecho, 7 (2): 23-50. DOI: 10.5354/0719-5885.2020.60562

Prieto, Alfredo, David Díaz, Isabel Lara, Jorge Montserrat, Paquita Sanvicen, Raúl Santiago, Alfredo Corell y Melchor Álvarez (2018). «Nuevas combinaciones de aula inversa con just in time teaching y análisis de respuestas de alumnos». RIED: Revista Iberoamericana de Educación a Distancia, 21 (1): 175-194. DOI: 10.5944/ ried.21.1.18836.

Rotellar, Cristina y Jeff Cain (2016). «Research, perspectives, and recommendations on implementingthe flipped classroom». American Journal of Pharmaceutical Education, 80 (2): 34. DOI: 10.5688/ajpe80234.

Salinas, Jesús (2020). «Educación en tiempos de pandemia: Tecnologías digitales en la mejora de los procesos educativos». Innovaciones Educativas, 22: 17-21. DOI: 10.22458/ie.v22iEspecial.3173.

SÁnchez, José, Julio Ruíz y Elena Sánchez (2017). «Flipped classroom: Claves para su puesta en práctica». EDMETIC, Revista de Educación Mediática y TIC, 6 (2): 336358. DOI: 10.21071/edmetic.v6i2.5832.

SÁnchez, María, Karen Urrejola y Nelson Becerra (2020). «Incorporación de dispositivos de enseñanza-aprendizaje de los géneros orales propios de la formación 
inicial en Derecho: El caso de la solemne oral». Revista Pedagogía Universitaria y Didáctica del Derecho, 7 (2): 51-74. DOI: 10.5354/0719-5885.2020.54883.

SÁnchez, Rut (2017). Aula invertida, metodología del siglo XXI. Islas Baleares: Universidad de las Islas Baleares.

Solari, Enzo (2012). «El currículo chileno de estudios». Revista de Derecho de la Pontificia Universidad Católica de Valparaíso, 39 (2): 703-734. Disponible en https:// bit.ly/2Yqui4g.

Tourón, Javier, Raúl Santiago y Alicia Díez (2014). The flipped classroom: Cómo convertir la escuela en un espacio de aprendizaje (Innovación educativa). Navarra: Océano.

\section{Sobre el autor}

Mauricio Figueroa es abogado. Candidato a doctor en la Universidad Castilla La Mancha, España, magíster en Derecho por la Universidad Católica del Norte, máster en Derecho Ambiental y Sostenibilidad por la Universidad de Castilla-La Mancha, España. Abogado, profesor de Historia y Geografía y licenciado en Educación de la Universidad de Tarapacá, diplomado en Educación Superior de la Universidad Santo Tomás. Docente de la Escuela de Derecho, Facultad de Derecho, Universidad Santo Tomás. Su correo electrónico es mfigueroa13@santotomas.cl. (D) https://orcid. org/0000-0002-1196-9904. 
La Revista Pedagogía Universitaria y Didáctica del Derecho (RPUDD) es una publicación científica semestral que contribuye a la reflexión multidisciplinaria sobre pedagogía universitaria y didáctica del derecho, para la formación y consolidación de esta área de investigación; así como a la difusión de prácticas innovadoras en la enseñanza-aprendizaje del derecho considerando el contexto nacional e internacional. Es una publicación electrónica internacional con una codirección entre Brasil y Chile.

\author{
DIRECTORA \\ María Francisca Elgueta Rosas \\ Universidad de Chile \\ DIRECTOR \\ Renato Duro Dias \\ Universidad Federal de Rio Grande, Brasil \\ SITIO WEB \\ pedagogiaderecho.uchile.cl \\ CORREO ELECTRÓNICO \\ rpedagogia@derecho.uchile.cl \\ LICENCIA DE ESTE ARTÍCULO
}

Creative Commons Atribución Compartir Igual 4.o Internacional

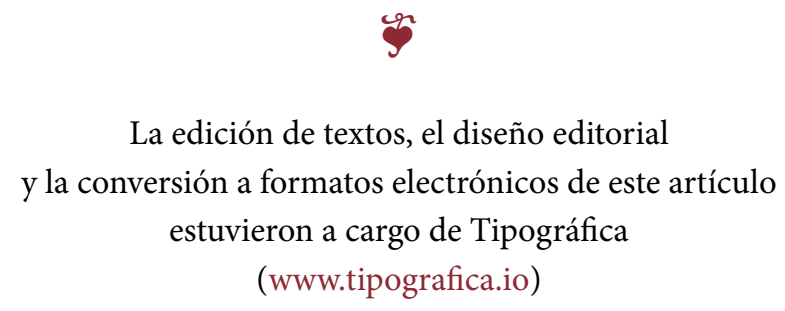

